\title{
Protective Effects of Purple Sweet Potato Added to Bacillus subtilis-Fermented Soymilk against Amyloid beta-Induced Memory Impairment
}

\author{
Ji Hyun Kim \\ Department of Food Science and Nutrition \\ Pusan National University \& Research Institute of Ecology for the Elderly \\ Busan 609-735, Korea \\ Tel: 82-51-510-2779Ｅ-mail: issun444@hanmail.net \\ Ji Myung Choi \\ Department of Food Science and Nutrition \\ Pusan National University \& Research Institute of Ecology for the Elderly \\ Busan 609-735, Korea \\ Tel: 82-51-510-2779_E-mail:poutia@naver.com \\ Sanghyun Lee \\ Department of Applied Plant Science, College of Industrial Science \\ Chung-Ang University, Anseong 456-756, Korea \\ Tel: 82-31-670-4688Ｅ-mail: slee@cau.ac.kr \\ Eun Ju Cho (Corresponding author) \\ Department of Food Science and Nutrition \\ Pusan National University \& Research Institute of Ecology for the Elderly \\ Busan 609-735, Korea \\ Tel: 82-51-510-2837 E-mail: ejcho@pusan.ac.kr
}

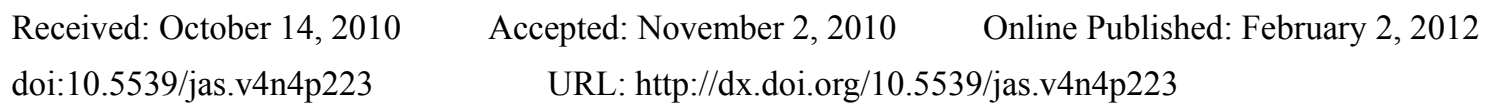

The research is funded by Ministry of Education and Science Technology (MEST) and the Korea Institute for Advancement of Technology (KIAT), Korea.

\begin{abstract}
The present study focused on the protective effects of 5\% purple sweet potato added to Bacillus subtilis-fermented soymilk (PFSM) under an Alzheimer's disease (AD) model using ICR mice induced by amyloid beta $(A \beta)$, which showed strong antioxidative activity against cognition impairment in a previous study. To observe memory and cognition abilities, T-maze, object cognition, and Morris water-maze tests were carried out. The A $\beta$ injected groups showed impairments of cognition and memory. However, the oral administration of PFSM extract (100 and $200 \mathrm{mg} / \mathrm{kg}$ body weight/day for 7 days) improved cognition and memory. Furthermore, nitric oxide (NO) formation and lipid peroxidation were significantly elevated by $A \beta$, whereas PFSM treatment significantly decreased NO formation and lipid peroxidation in the brain, liver, and kidneys. The present study suggests that PFSM improves memory deficit and cognition ability following A $\beta$ induction. The protective
\end{abstract}


effects of PFSM against A $\beta$-induced impairments of memory and cognition are closely related to the attenuation of oxidative stress.

Keywords: Amyloid beta, Alzheimer's disease, Memory, Oxidative stress, Soymilk

\section{Introduction}

Oxidative stress from free radicals has been thought to play an important role in age-related neurodegenerative disorders (Harman, 1995). Free radical-mediated damage to neuronal membrane components has been implicated in the etiology of diseases of aging such as Alzheimer's disease (AD) (Butterfield and Lauderback, 2002). $\mathrm{AD}$, the most common cause of dementia in the elderly, is an age-dependent progressive neurodegenerative disorder that results in global impairment of higher mental functions, memory loss, and other cognitive impairments, as well as changes in personality and behavior (Palmer, 2002).

Among the theories on causes of $\mathrm{AD}$, it is well accepted that the overproduction and aggregation of amyloid- $\beta$ $(\mathrm{A} \beta)$ is the most important risk factor. The accumulation of $\mathrm{A} \beta$ in the brain is associated with progressive neuronal death and cognitive deficits (Olariu et al., 2002; Hashimoto et al., 2002). The deposition of A $\beta$ is invariably associated with oxidative stress and inflammatory responses that contribute to neuronal dysfunction or death (Butterfield et al., 2007). Therefore, prevention of neurotoxicity induced by A $\beta$ is the most crucial strategy for treatment of AD.

Many studies suggest the possible therapeutic uses of free radical scavengers and antioxidants in the treatment of AD pathogenesis (Pappolla et al., 1997; Bruce et al., 1996). Soybeans, the most common legume around the world, are abundant in high quality protein and antioxidant activity. Although they are rich in quality, soybeans have some limitations such as a characteristic bean flavor, and problems of digestion by humans due to raffinose and stachyose (Thananunkul et al., 1976). To overcome these problem and to develop functional probiotics, we manufactured soymilk (SM) fermented by Bacillus subtilis isolated from Cheonggukjang (Choi et al., 2010). Fermentations are characterized by extensive hydrolysis of soybean proteins to amino acids, peptides, and ammonia, resulting in improvements of functionality (Steinkraus, 1997). Fermented soymilk (FS), unlike other fermented milks or yogurt drinks, contains no lactose or cholesterol, and is considered to have health benefits from both the soy itself and fermentation by Bacillus subtilis. SM fermented by Bacillus subtilis isolated from Cheonggukjang may lead to a combination of benefits as a probiotic and from the transformation of isoflavone glucosides to bioactive isoflavone aglycones (Choi et al., 2010). In the present study, we manufactured functional FS using Bacillus subtilis with the addition of purple sweet potato hot water extract (PSPE) to enhance taste and activity. Its protective role against $\mathrm{AD}$ was then investigated under an $\mathrm{A} \beta_{25-35}$-injected animal model.

\section{Materials and Methods}

\subsection{Reagents}

The soymilk and purple sweet potato hot water extract (PSPE) were provided by Chunho Foods (Busan, Korea). Bacillus subtilis 2829 PNU-015 was isolated from Cheonggukjang that was acquired in Sunchang Province, Korea. $\mathrm{A} \beta_{25-35}$ and malondialdehyde (MDA) were obtained from Sigma Aldrich (Saint Louis, Missouri, USA). Dimethyl sulfoxide (DMSO) and sodium chloride $(\mathrm{NaCl})$ were purchased from Bio Basics Inc. (Ontaio, Canada). Thiobarbituric acid (TBA) was provided by Lancaster Synthesis (Ward Hill, USA). Phosphoric acid and 1-butanol were acquired from Samchun Pure Chemical Co. (Gyeonggi, Pyeongtaek, Korea).

2.2 Preparation of Methanol (MeOH) Extracts of 5\% Purple Sweet Potato Added to Bacillus Subtilis-fermented Soymilk (PFSM)

The SM preparation method was as follows. One percent glucose was added to the SM, which was then autoclaved at $121^{\circ} \mathrm{C}$ for $15 \mathrm{~min}$ and cooled. Bacillus subtilis 2829 PNU-015 was inoculated into the SM, incubated in a shaking water bath until pH 5.5 was reached, and then the PSPE was added. For the experiments, freeze-dried PFSM was extracted by $\mathrm{MeOH}$ at room temperature for 24 hours and the $\mathrm{MeOH}$ extraction process was repeated 3 times. The extract was concentrated using a rotary evaporator and dissolved in DMSO.

\subsection{Animals and Experimental Protocols}

Male ICR mice (5 weeks olds, Orient Inc. Seongnam, Korea) weighing 25-27 g were housed in plastic cages with free access to food and water, and were maintained in a controlled environment $\left(20 \pm 2^{\circ} \mathrm{C}, 50 \pm 10 \%\right.$, 12-hour light/dark cycle). The animal protocol used in this study was reviewed and approved (approval number PNU-2009-0044) by the Pusan National University-Institutional Animal Care and Use Committee (PNU-IACUC) on the ethical procedures and scientific care of laboratory animals. Five-week-old ICR mice were 
divided into four groups. There were no significant differences in body weight among the groups helping to eliminate physical differences due to body weight variation. The normal group and control group were orally given water (each group, $\mathrm{n}=8$ ), and the other two groups were administered PFSM orally at doses of 100 and $200 \mathrm{mg} / \mathrm{kg} /$ day for 7 days (each group, $\mathrm{n}=8$ ) using zonde. The behavioral procedures are shown in Fig. 1 .

\subsection{A $\beta_{25-35}$ Infused Mouse Model}

The $\mathrm{A} \beta_{25-35}$ was dissolved and diluted in saline solution $(0.9 \% \mathrm{NaCl})$ at a concentration of $5 \mathrm{nmol}$. The dissolved $\mathrm{A} \beta_{25-35}$ was incubated for aggregation at $37^{\circ} \mathrm{C}$ for 3 days before injection. Saline solution containing aggregated $\mathrm{A} \beta_{25-35}$ was injected according to the procedure established by Laursen and Belknap (1986). The mice were lightly anesthetized with ether, and injected at bregma using a $10 \mu \mathrm{L}$ Hamilton microsyringe fitted with a 26 gauge needle that was inserted to a depth of $2.2 \mathrm{~mm}$. The volume of the injection was $5 \mu \mathrm{L}(5 \mathrm{nmol} / \mathrm{mouse})$.

\subsection{Novel Object Recognition Test}

The object recognition test (Bevins and Besheer, 2006) was performed in a square open-field apparatus $(40 \times 30$ $\times 20 \mathrm{~cm}$ ) painted black in color. Two identical objects (plastic bottles) were placed at a fixed distance within a square field. The mice were placed at the center of the square field, and the number of touch times of each object was recorded during a 10 min period (training session). The mice were placed back into the same field 24 hours after the training session, in which one of the old objects used during the training session was replaced with a new object (another plastic bottle). The mice were allowed to search freely for $10 \mathrm{~min}$, and the number of touch times was recorded (test session).

\subsection{T-maze Test}

The T-maze test was conducted according to the procedure established by Montgomery (1952). This apparatus was T-shaped, the walls of the maze were made of black painted board (length of start and goal stems $=50 \mathrm{~cm}$, width $=13 \mathrm{~cm}$, height $=20 \mathrm{~cm}$ ) and were glued to a black square board bottom piece. The apparatus consisted of a start box, left arm, and right arm with a block door that could be separated. The mice were placed at the start box, and the number of touch and exploration times was recorded during a $10 \mathrm{~min}$ period (training session). The mice were placed back into the same apparatus 24 hours after the training session. The mice were allowed to search freely for $10 \mathrm{~min}$, and the number of touch and exploration times was recorded (test session).

\subsection{Morris Water Maze Test}

The Morris water maze test was conducted according to the procedure established by Morris (1984) with slight modification. The apparatus used in the Morris water maze test consisted of a dark plastic circular pool $80 \mathrm{~cm}$ in diameter and surrounded by a $40 \mathrm{~cm}$ high wall, randomly divided into quadrants. Milk powder was put into the pool to make the water opaque. The water temperature was maintained at $22 \pm 1^{\circ} \mathrm{C}$. A platform $(8 \mathrm{~cm}$ diameter $)$ was placed $1 \mathrm{~cm}$ below the water surface in the middle of one quadrant. The position of the platform was unchanged during the training session. Visual cues for navigation were provided by four posters on the walls of the apparatus. Three training trials per day were conducted for 3 days. In training trials for the Morris water maze test, the mice were randomly placed in the water facing the pool wall and allowed to swim for a maximum of $60 \mathrm{~s}$. The latency to find the platform was recorded. The mice that found the platform were allowed to take a rest on the platform for $15 \mathrm{~s}$. If a mouse could not find the platform within $60 \mathrm{~s}$, it was guided by an experimenter to find the platform and allowed to stay on it for $15 \mathrm{~s}$. A probe trial of the Morris water maze test was performed on the day after 3 days of training was finished. In the primary test, the experiment was performed the same as before. For the secondary test, it was performed without the platform. The mice were placed in the pool and swam for $60 \mathrm{~s}$ looking for the platform, and the latency time that the mice stayed at the position of the first platform was recorded. In a tertiary test, the water was transparent and the number of times to reach the platform was counted.

\subsection{Measurement of Lipid Peroxidation}

MDA levels were measured by the method of Ohkawa et al. (1979). After completion of the behavioral observations, the mice in every group were anesthetized by ether. The brain, liver, and kidneys were removed immediately and placed on ice. The dissected tissue was homogenized with saline solution. This homogenate was mixed with $1 \%$ phosphoric acid and $0.67 \%$ TBA solution. This solution mixture was refrigerated in an ice after boiling for $45 \mathrm{~min}$ and then $2 \mathrm{~mL}$ of 1-butanol was added, followed by centrifugation of the mixture at 3000 rpm for $10 \mathrm{~min}$. The absorbance values of the supernatant were measured at $535 \mathrm{~nm}$ and $520 \mathrm{~nm}$. The yield of lipid peroxidase was calculated with the standard curve of MDA content. 


\subsection{Nitric Oxide (NO) Scavenging Activity}

The NO concentrations of tissues were determined by the method of Schmidt et al. (1992). One-hundred fifty microliters of the supernatant used in the lipid peroxidation method was mixed with $130 \mu \mathrm{L}$ of distilled water, and then $20 \mu \mathrm{L}$ of the mixed solution was added to the same amount of phosphoric acid and $0.1 \%$ $\mathrm{N}$-(1-naphthyl) ethylenediamide dihydrochloride solution. The absorbance value of the mixture was then measured at $540 \mathrm{~nm}$. The yield of NO production was calculated with a standard curve of sodium nitrite $\left(\mathrm{NaNO}_{2}\right)$ content.

\subsection{Statistical Analysis}

All statistical analyses were performed with SAS software (SAS Institute, Cary, NC, USA). $P<0.05$ was deemed statistically significant. The measurement data are expressed as means \pm standard deviations.

\section{Results}

\subsection{Novel Object Recognition Task}

Figure 2 shows the results of the object recognition test. After 24 hours of training, cognition ability toward the old object was not significantly different among the groups. On the other hand, cognition ability toward the new object was lower in groups injected with $\mathrm{A} \beta_{25-35}$ as compared to the normal group. The groups administered PFSM at doses of 100 and $200 \mathrm{mg} / \mathrm{kg} /$ day for 7 days revealed significantly greater numbers of touches and spending times toward the novel object than the old object during the test session, indicating that PFSM improved recognition ability in mice impaired by $\mathrm{A} \beta_{25-35}$ infusion.

\subsection{T-maze Test}

The PFSM was administered orally every day for 1 week to the mice induced by $A \beta_{25-35}$, and then the T-maze test was conducted on the day after the end of the novel object recognition test. Figure 3 shows the percentage of new route cognition in all mice groups. The control group showed lower spatial perception ability toward the new route compared to the normal group. However, cognition ability toward the new route by mice treated with PFSM (100 and $200 \mathrm{mg} / \mathrm{kg} /$ day) was higher than in the control group.

\subsection{Morris Water Maze Task}

The results of the Morris water maze test are shown in Fig. 4, 5, and 6. Although the latency to reach the platform decreased in all groups by training, the longest time to reach the platform was observed in the control group (Fig. 4). Moreover, the latencies of the normal and PFSM treated groups were shorter than that of the control group by repeating training for 3 days. Figure 5 indicates the occupancy time of each experimental group in the zone containing the cue poster when the platform was removed. The time spent on the platform and the memory ability of the position were shorter in the control group compared to the normal group, whereas the administration of PFSM improved the memory ability of the position, showing longer time spent on the platform. The results of working ability are indicated in Fig. 6. There were no significant differences in mean latency to find the exposed platform among all groups of mice in the probe trial. This result supports that the protective role of PFSM from cognition ability impairments due to $\mathrm{A} \beta_{25-35}$, is not associated with exercise and visual abilities.

\subsection{Measurement of Lipid Peroxidation}

Table 1 shows the inhibitory effects of PFSM against lipid peroxidation induced by $\mathrm{A} \beta_{25-35}$. The control group had significantly increased MDA levels in all tissues including the brain, liver and kidneys compared to the normal group. MDA levels decreased significantly in the groups administered PFSM at doses of 100 and 200 $\mathrm{mg} / \mathrm{kg} /$ day and the decreases were oral-dose dependent.

\subsection{NO Scavenging Activity}

The protective effects of PFSM against NO production are shown in Table 2. In comparison with the normal group, NO values in the brain, liver and kidneys were significantly elevated in the control group. However, the administration of PFSM at 100 and $200 \mathrm{mg} / \mathrm{kg} /$ day doses led to decreases in NO levels oral dose-dependently. In particular, NO scavenging activity was higher in the brain than the other tissues.

\section{Discussion}

Oxidative stress induced by $\mathrm{A} \beta$ leads to toxic inflammatory responses, and eventually results in the pathological conditions of AD (Markesbery, 1997; Yankner, 1996). Treatment of AD, which has been estimated to account for $50 \%-60 \%$ of dementia cases in persons over 65 years of age (Ritchie and Lovestone, 2002), is now becoming a social problem. Although numerous possible drugs for $\mathrm{AD}$ exist, such as donepezil, rivastigmine, and galantamine (Bartolini et al., 2003; De Ferrari et al., 2001), the effort to discover a diet of natural foods with 
abundant antioxidant activity for prevention of $\mathrm{AD}$ are particularly important, because many free radical scavengers and antioxidant substances such as vitamin E, vitamin C, flavonoids, and carotenoids have no serious side effects (Bastianetto et al., 2000).

The AD model induced by injection of $\mathrm{A} \beta$ using ICR mice is well established for the study of AD pathology. The brain is especially sensitive to oxidative stress due to its high concentration of quickly oxidizable fatty acids and high oxygen consumption (Selkoe, 2006; Abdul et al., 2008). Several reports have demonstrated that intracerebroventricular administration of $\mathrm{A} \beta$ into the brain induces histological and biochemical changes, memory deficits, oxidative stress, and inflammatory responses (Maurice et al., 1996; Olariu et al., 2001). Therefore, oxidative stress from free radicals induced by $A \beta$ and the transformation of $A \beta$ to aggregate forms are states of crucial importance for generating toxic effects. These toxic effects consequently lead to many neurodegenerative disorders, in particular AD (Walsh and Selkoe, 2004). Thus, the injection of A $\beta$ into the bregma of ICR mice brains results in pathological symptoms, deficits in learning and memory, and neurodegeneration. In particular, $A \beta_{25-35}$ is the shortest peptide sequence that maintains biological activity comparable to that of full-length $\mathrm{A} \beta$ peptides, namely $\mathrm{A} \beta$ peptides 1-42 (A $\left.\beta_{1-42}\right)$ (D'Ursi et al., 2004). $\mathrm{A} \beta_{25-35}$ has been used to investigate the impairment of learning and memory, since it is a less expensive and an easily influential substitute for $A \beta_{1-42}$. Several studies have indicated that $A \beta_{25-35}$ is more toxic to cultured neurons, exhibits toxicity earlier, causes more oxidation in protein and cell membranes, and aggregates faster than $A \beta_{1-42}$ (Varadarajan et al., 2001; Choi et al., 2003; Trubetskaya et al., 2003; Atwood et al., 1998). For these reasons, $\mathrm{A} \beta_{25-35}$ injection in mice is effective for examining improvements of cognitive impairment. We previously reported that PFSM showed radical scavenging activity and attenuated oxidative stress induced by free radicals under a cellular system (Harman, 1995). Therefore, in the present study, we focused on protective effects from cognitive dysfunction using an $A \beta_{25-35}$-injected animal model of AD. Novel object recognition, T-maze, and Morris water maze tests were carried out. Moreover, inhibition effects against lipid peroxidation as well as NO scavenging activity were also investigated.

Numerous studies have demonstrated the beneficial effects of FS on health, including antioxidant activity and anti-inflammation effects (Wang et al., 2006; Lin et al., 2005; Kwak et al., 2007; Liu et al., 2009). In addition, purple sweet potato (PSP) is known to have protective effects on neuronal cells (Lu et al., 2010). Therefore, we attempted to manufacture functional FS with additions of PSP, and then protective effects against $\mathrm{A} \beta_{25-35}$-induced cognitive dysfunction and neurotoxicity by PFSM were observed. In the present study, PFSM significantly ameliorated cognitive impairments induced by $\mathrm{A} \beta_{25-35}$ infusion in the novel object recognition, T-maze, and Morris water maze tests. In the novel object recognition test, the difference in exploration between a previously seen object and a novel object is taken as an index of memory performance. The test offers the possibility to obtain rapid information on the amnesiant potential of functional substances, and thus can be considered a useful tool to investigate learning and memory function effectively (Ennaceur and Delacour, 1988). The present results show that percentages of novel object recognition were higher in groups administered PFSM than in the control group, suggesting improvements of cognition ability. In addition, the T-maze test examines the short-term memory of mice. The number of entries and touch times in a new route were recorded. In the groups administered PFSM, the number of entries into a new route was significantly higher than in the control group (Gerlai, 1998). The results of the two aforementioned behavioral tests indicate that PFSM protected against impairments of learning and memory function in mice induced by $\mathrm{A} \beta_{25-35}$.

The Morris water maze is used to test spatial cognition ability and long-term memory (Morris, 1984). For the mice in all groups, latency to find the platform was shortened by the training trial. Compared with the control group, the groups administered PFSM showed shorter times to reach the platform and longer times spent at the position, indicating memory improvement ability by PFSM. Furthermore, the time to reach the exposed platform was not significantly different among the groups. There results indicate that protective effects from PFSM deficits of long-term spatial cognition induced by $\mathrm{A} \beta_{25-35}$ are not involved in swimming or visual ability. Taken together, PFSM may ameliorate dysfunctions of learning and memory caused by $\mathrm{A} \beta_{25-35}$.

$\mathrm{A} \beta_{25-35}$ induces oxidative stress from the over-production of free radicals, which cause peroxidation of proteins and lipids in neurons. Based on this, lipid peroxidation is a central mechanism of neurodegeneration in $\mathrm{AD}$ (Palmer and Burns, 1994; Markesbery and Lovell, 1998). MDA is an end product of lipid peroxidation, therefore it is used as an indicator of lipid peroxidation. The injection of $A \beta_{25-35}$ induces a notable increase of MDA content in the brain hippocampus, indicating that $A \beta_{25-35}$ induces neuronal lipid peroxidation (Fang and Liu, 2006; Feng et al., 2005). Furthermore, several studies suggest that NO may be directly or indirectly involved in neuronal death in AD and other neurodegenerative disorders (Bredt, 1999; Dawson and Dawson, 1998; Kaufman, 1999). From the present results, we can confirm that PFSM significantly decreased MDA content and NO 
radicals in tissues such as the liver, brain, and kidneys of mice injected with $A \beta_{25-35}$. In particular, protective effects of PFSM against lipid peroxidation and NO production were greater in the brain than other tissues.

The present study shows that the injection of $A \beta_{25-35}$ caused significant impairments of memory and learning ability, and resulted in oxidative stress. However, the oral administration of PFSM significantly protected against learning and memory deficits induced by $\mathrm{A} \beta_{25-35}$. Furthermore, PFSM inhibited lipid peroxidation and NO production, suggesting a protective role from oxidative stress by $A \beta_{25-35}$. The present study indicates the promising role of PFSM as an agent for AD treatment.

\section{Conclusion}

The administration of PFSM improved deficits of learning and memory ability by protecting against oxidative stress induced by $\mathrm{A} \beta_{25-35}$. Furthermore, NO formation and lipid peroxidation were significantly elevated by $\mathrm{A} \beta$, whereas PFSM treatment significantly decreased NO formation and lipid peroxidation in the brain, liver, and kidneys. The protective effects of PFSM against A $\beta$-induced impairments of memory and cognition ability are closely related to the attenuation of oxidative stress. The present study supports the possibility of functional SM products with antioxidative activity that can improve cognition and memory abilities.

\section{References}

Abdul, H. M., Sultana, R., St Clair, D. K., Markesbery, W. R., \& Butterfield, D. A. (2008). Oxidative damage in brain from human mutant APP/PS-1 double knock-in mice as a function of age. Free Radical Biology and Medicine, 45, 1420-1425.

Atwood, C. S., Moir, R. D., Huang, X., Scarpa, R. C., Bacarra, N. M. E., Romano, D. M., Hartshorn, M. A., Tanzi, R. E. \& Bush, A. I. (1998). Dramatic aggregation of Alzheimer A $\beta$ by $\mathrm{Cu}(\mathrm{II})$ is induced by conditions representing physiological acidosis. The Journal of Biological Chemistry, 273, 12817-12826.

Bartolini, M., Bertucci, C., Cavrini, V., \& Andrisano, V. (2003). $\beta$-Amyloid aggregation induced by human acetylcholinesterase: inhibition studies. Biochemical Pharmacology, 65, 407-416.

Bastianetto, S., Ramassamy, C., Doré, S., Christen, Y., Poirier, J., \& Quirion, R. (2000). The ginkgo biloba extract (EGb 761) protects hippocampal neurons against cell death induced by $\beta$-amyloid. The European Journal of Neuroscience, 12, 1882-1890.

Bevins, R. A., \& Besheer, J. (2006). Object recognition in rats and mice: a one-trial non-matching-to-sample learning task to study 'recognition memory'. Nature Protocols, 1, 1306-1311.

Bredt, D. S. (1999). Endogenous nitric oxide synthesis: biological functions and pathophysiology. Free Radical Research, 31, 577-596.

Bruce, A. J., Malfroy, B., \& Baudry, M. (1996). $\beta$-Amyloid toxicity in organotypic hippocampal cultures: protection by EUK-8, a synthetic catalytic free radical scavenger. Proceedings of the National Academy of Sciences of the United States of America, 93, 2312-2316.

Butterfield, D. A., \& Lauderback, C. M. (2002). Lipid peroxidation and protein oxidation in Alzheimer's disease brain: potential causes and consequences involving amyloid $\beta$-peptide-associated free radical oxidative stress. Free Radical Biology and Medicine, 32, 1050-1060.

Butterfield, D. A., Reed, T., Newman, S. F., \& Sultana, R. (2007). Roles of amyloid $\beta$-peptide-associated oxidative stress and brain protein modifications in the pathogenesis of Alzheimer's disease and mild cognitive impairment. Free Radical Biology and Medicine, 43, 658-677.

Choi, J. M., Kim, J. H., \& Cho, E. J. (2010). Protective activity of purple sweet potato extract-added soymilk fermented by Bacillus subtilis against oxidative stress. Food Science and Biotechnology, 19, 457-462

Choi, J., Malakowsky, C. A., Talent, J. M., Conrad, C. C., Carroll, C. A., Weintraub, S. T., \& Gracy, R. W. (2003). Anti-apoptotic proteins are oxidized by $\mathrm{A} \beta_{25-35}$ in Alzheimer's fibroblasts. Biochimica et Biophysica Acta, 1637, 135-141.

Dawson, V. L., \& Dawson, T. M. (1998). Nitric oxide in neurodegeneration. Progress in Brain Research, 118, 215-229.

De Ferrari, G. V., Canales, M. A., Shin, I., Weiner, L. M., Silman, I., \& Inestrosa, N. C. (2001). A structural motif of acetylcholinesterase that promotes amyloid $\beta$-peptide fibril formation. Biochemistry, 40, 10447-10457.

D’Ursi, A. M., Armenante, M. R., Guerrini, R., Salvadori, S., Sorrentino, G., \& Picone, D. (2004). Solution structure of amyloid $\beta$-peptide (25-35) in different media. Journal of Medicinal Chemistry, 47, 4231-4238. 
Ennaceur, A., \& Delacour, J. (1988). A new one-trial test for neurobiological studies of memory in rats. 1: Behavioral data. Behavioral Brain Research, 31, 47-59.

Fang, F., \& Liu, G. T. (2006). Protective effects of compound FLZ on $\beta$-amyloid peptide-(25-35)-induced mouse hippocampal injury and learning and memory impairment. Acta Pharmacologica Sinica, 27, 651-658.

Feng, W., Wei, H., \& Liu, G. T. (2005). Pharmacological study of the novel compound FLZ against experimental Parkinson's models and its active mechanism. Molecular Neurobiology, 31, 295-300.

Gerlai, R. (1998). A new continuous alternation task in T-maze detects hippocampal dysfunction in mice. A strain comparison and lesion study. Behavioral Brain Research, 95, 91-101.

Harman, D. (1995). Free radical theory of aging: Alzheimer's disease pathogenesis. Age, 18, 97-119.

Hashimoto, M., Hossain, S., Shimada, T., Sugioka, K., Yamasaki, H., Fujii, Y., Ishibashi, Y., Oka, J., \& Shido, O. (2002). Docosahexaenoic acid provides protection from impairment of learning ability in Alzheimer's disease model rats. Journal of Neurochemistry, 81, 1084-1091.

Kaufman, P. L. (1999). Nitric-oxide synthase and neurodegeneration/neuroprotection. Proceedings of the National Academy of Sciences of the United States of America, 96, 9455-9456.

Kwak, C. S., Lee, M. S., \& Park, S. C. (2007). Higher antioxidant properties of Chungkookjang, a fermented soybean paste, may be due to increased aglycone and malonylglycoside isoflavone during fermentation. Nutrition Research, 27, 719-727.

Laursen, S. E., \& Belknap, J. K. (1986). Intracerebroventricular injections in mice. Some methodological refinements. Journal of Pharmacological Methods, 16, 355-357.

Liu, C. F., Hu, C. L., Chiang, S. S., Tseng, K. C., Yu, R. C., \& Pan, T. M. (2009). Beneficial preventive effects of gastric mucosal lesion for soy-skim milk fermented by lactic acid bacteria. Journal of Agricultural and Food Chemistry, 57, 4433-4438.

Lin, C. Y., Tsai, Z. Y., Cheng, I. C., \& Lin, S. H. (2005). Effects of fermented soy milk on the liver lipids under oxidative stress. World Journal of Gastroenterology, 11, 7355-7358.

Lu, J., Wu, D. M., Zheng, Y. L., Hu, B., \& Zhang, Z. F. (2010). Purple sweet potato color alleviates D-galactose-induced brain aging in old mice by promoting survival of neurons via PI3K pathway and inhibiting cytochrome C-mediated apoptosis. Brain Pathology, 20, 598-612.

Markesbery, W. R. (1997). Oxidative stress hypothesis in Alzheimer's disease. Free Radical Biology and Medicine, 23, 134-147.

Markesbery, W. R., \& Lovell, M. A. (1998). Four-hydroxynonenal, a product of lipid peroxidation, is increased in the brain in Alzheimer's disease. Neurobiology of Aging, 19, 33-36.

Maurice, T., Lockhart, B. P., \& Privat, A. (1996). Amnesia induced in mice by centrally administered $\beta$-amyloid peptides involves cholinergic dysfunction. Brain Research, 706, 181-193.

Montgomery, K. C. (1952). A test of two explanations of spontaneous alternation. Journal of Comparative and Physiological Psychology, 45, 287-293.

Morris, R. (1984). Developments of a water-maze procedure for studying a spatial learning in the rat. Journal of Neuroscience Methods, 11, 47-60.

Ohkawa, H., Ohishi, N., \& Yagi, K. (1979). Assay for lipid peroxides in animal tissues by thiobarbituric acid reaction. Analytical Biochemistry, 95, 351-358.

Olariu, A., Tran, M. H., Yamada, K., Mizuno, M., Hefco, V., \& Nabeshima, T. (2001). Memory deficits and increased emotionality induced by $\beta$-amyloid (25-35) are correlated with the reduced acetylcholine release and altered phorbol dibutyrate binding in the hippocampus. Journal of Neural Transmission, 108, 1065-1079.

Olariu, A., Yamada, K., Mamiya, T., Hefco, V., \& Nabeshima, T. (2002). Memory impairment induced by chronic intracerebroventricular infusion of beta-amyloid (1-40) involves downregulation of protein kinase C. Brain Research, 957, 278-286.

Palmer, A. M. (2002). Pharmacotherapy for Alzheimer's disease: progress and prospects. Trends in Pharmacological Sciences, 23, 426-433.

Palmer, A. M., \& Burns, M. A. (1994). Selective increase in lipid peroxidation in the inferior temporal cortex in Alzheimer's disease. Brain Research, 645, 338-342. 
Pappolla, M. A., Sos, M., Omar, R. A., Bick, R. J., Hickson-Bick, D. L., Reiter, R. J., Efthimiopoulos, S., \& Robakis, N. K. (1997). Melatonin prevents death of neuroblastoma cells exposed to the alzheimer amyloid peptide. The Journal of Neuroscience, 17, 1683-1690.

Ritchie, K., \& Lovestone, S. (2002). The dementias. The Lancet, 360, 1759-1766.

Schmidt, H. H., Warner, T. D., Nakane, M., Förstermann, U., \& Murad, F. (1992). Regulation and subcellular location of nitrogen oxide synthases in RAW264.7 macrophages. Molecular Pharmacology, 41, 615-624.

Selkoe, D. J. (2006). Toward a comprehensive theory for Alzheimer's disease. Hypothesis: Alzheimer's disease is caused by the cerebral accumulation and cytotoxicity of amyloid $\beta$-protein. Annals of the New York Academy of Sciences, 924, 17-25.

Steinkraus, K. H. (1997). Classification of fermented foods: worldwide review of household fermentation techniques. Food Control, 8, 311-317.

Thananunkul, D., Tanaka, M., Chichester, C. O., \& Lee, T. C. (1976). Degradation of raffinose and stachyose in soybean milk by $\alpha$-galactosidase from Mortierella vinacea. Entrapment of $\alpha$-galactosidase within polyacrylamide gel. Journal of Food Science, 41, 173-175.

Trubetskaya, V. V., Stepanichev, M. Y., Onufriev, M. V., Lazareva, N. A., Markevich, V. A., \& Gulyaeva, N. V. (2003). Administration of aggregated beta-amyloid peptide (25-35) induces changes in long-term potentiation in the hippocampus in vivo. Neuroscience and Behavioral Physiology, 33, 95-98.

Varadarajan, S., Kanski, J., Aksenova, M., Lauderback, C., \& Butterfield, D. A. (2001). Different mechanisms of oxidative stress and neurotoxicity for Alzheimer's $A \beta(1-42)$ and $A \beta(25-35)$. Journal of the American Chemical Society, 123, 5625-5631.

Walsh, D. M., \& Selkoe, D. J. (2004). Deciphering the molecular basis of memory failure in Alzheimer's disease. Neuron, 44, 181-193.

Wang, Y. C., Yu, R. C., \& Chou, C. C. (2006). Antioxidative activities of soymilk fermented with lactic acid bacteria and bifidobacteria. Food Microbiology, 23, 128-135.

Yankner, B. A. (1996). Mechanisms of neuronal degeneration in Alzheimer's disease. Neuron, 16, 921-932.

Table 1. Protective activity of extract from purple sweet potato added to Bacillus subtilis-fermented soymilk against lipid peroxidation in mice induced by $\mathrm{A} \beta_{25-35}$

\begin{tabular}{|c|c|c|c|}
\hline \multirow{2}{*}{ Group } & \multicolumn{3}{|c|}{ MDA (nmole/mg protein) } \\
\cline { 2 - 4 } & Brain & Liver & Kidney \\
\hline Normal & $12.70 \pm 1.18^{\mathrm{b}}$ & $18.89 \pm 1.23^{\mathrm{ab}}$ & $5.39 \pm 0.66^{\mathrm{b}}$ \\
\hline Control & $43.01 \pm 4.15^{\mathrm{a}}$ & $27.95 \pm 8.03^{\mathrm{a}}$ & $9.74 \pm 0.93^{\mathrm{a}}$ \\
\hline PFSM (100) & $11.10 \pm 0.48^{\mathrm{b}}$ & $19.55 \pm 1.86^{\mathrm{ab}}$ & $9.21 \pm 0.46^{\mathrm{a}}$ \\
\hline PFSM (200) & $9.32 \pm 1.30^{\mathrm{b}}$ & $12.85 \pm 2.15^{\mathrm{b}}$ & $5.88 \pm 0.64^{\mathrm{b}}$ \\
\hline
\end{tabular}

Values are means \pm SD. ${ }^{\text {a-b }}$ Means with different letters are significantly different $(p<0.05)$ by Duncan's multiple range test. Normal group, $0.9 \% \mathrm{NaCl}$ injection + oral administration of water; Control group, A $\beta 25-35$ injection + oral administration of water; PFSM(100) group, Aß25-35 injection + Oral administration of PFSM MeOH extract (100 mg/kg/day); PFSM(200) group, A $325-35$ injection + Oral administration of PFSM MeOH extract (200 $\mathrm{mg} / \mathrm{kg} /$ day).

Table 2. Effects of oral administration of extract from purple sweet potato added to Bacillus subtilis-fermented soymilk on $\mathrm{A} \beta_{25-35}$-induced nitric oxide formation

\begin{tabular}{|c|c|c|c|}
\hline \multirow{2}{*}{ Group } & \multicolumn{3}{|c|}{$\mathrm{NaNO}_{2}(\mu \mathrm{mol} / \mathrm{L} / \mathrm{mg}$ protein $)$} \\
\cline { 2 - 4 } & Brain & Liver & Kidney \\
\hline Normal & $23.49 \pm 1.49^{\mathrm{b}}$ & $79.51 \pm 4.31^{\mathrm{b}}$ & $32.12 \pm 1.28^{\mathrm{ab}}$ \\
\hline Control & $29.95 \pm 1.51^{\mathrm{a}}$ & $93.23 \pm 1.16^{\mathrm{a}}$ & $35.50 \pm 0.92^{\mathrm{a}}$ \\
\hline PFSM (100) & $23.18 \pm 1.18^{\mathrm{b}}$ & $84.25 \pm 3.07^{\mathrm{b}}$ & $32.04 \pm 3.29^{\mathrm{ab}}$ \\
\hline PFSM (200) & $14.81 \pm 0.8^{\mathrm{b}}$ & $71.73 \pm 4.38^{\mathrm{b}}$ & $27.17 \pm 1.7^{\mathrm{b}}$ \\
\hline
\end{tabular}

Values are means \pm SD. ${ }^{\text {a-b }}$ Means with different letters are significantly different $(p<0.05)$ by Duncan's multiple range test. Abbreviations are shown in Table 1. 


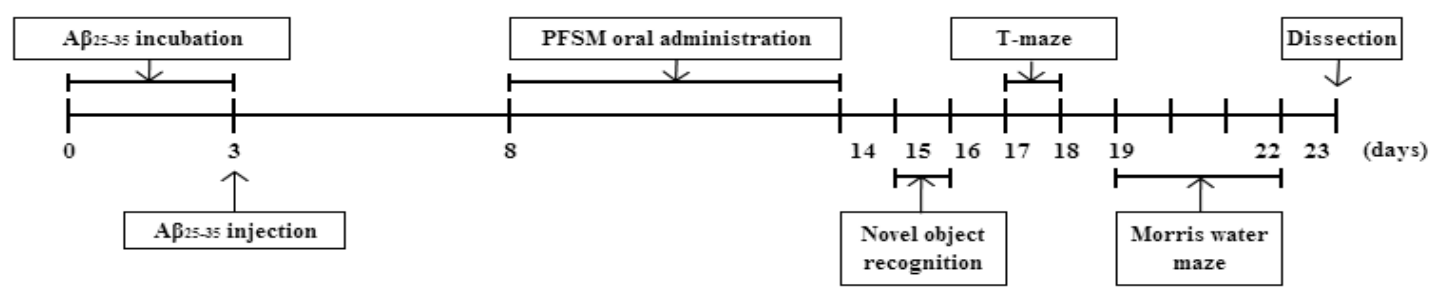

Figure 1. Behavioral experimental schedule for mice injected $A \beta_{25-35}$

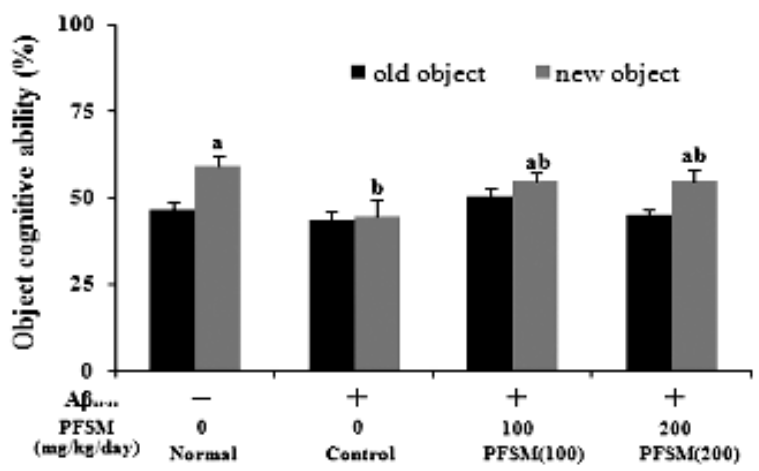

Figure 2. Effects of extract from purple sweet potato added to Bacillus subtilis-fermented soymilk in object recognition tests. Values are mean $\pm \mathrm{SD}$. ${ }^{\mathrm{a}-\mathrm{b}}$ Means with different letters are significantly different $(p<0.05)$ by Duncan's multiple range test. Abbreviations are shown in Table 1

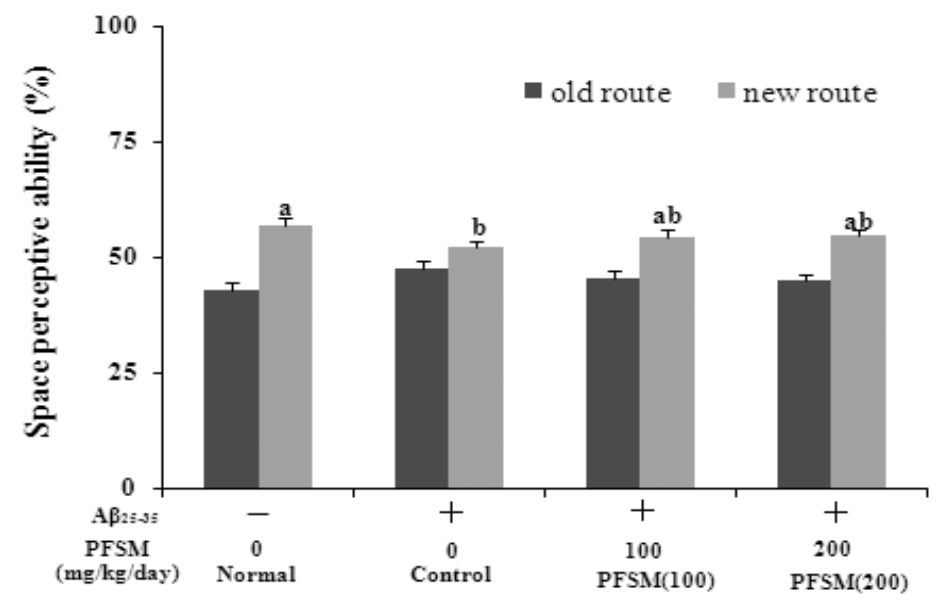

Figure 3. Effects of extract from purple sweet potato added to Bacillus subtilis-fermented soymilk on spatial alternation tests in a T-maze. Values are mean \pm SD. ${ }^{\text {a-b }}$ Means with different letters are significantly different $(p$ $<0.05$ ) by Duncan's multiple range test. Abbreviations are shown in Table 1 


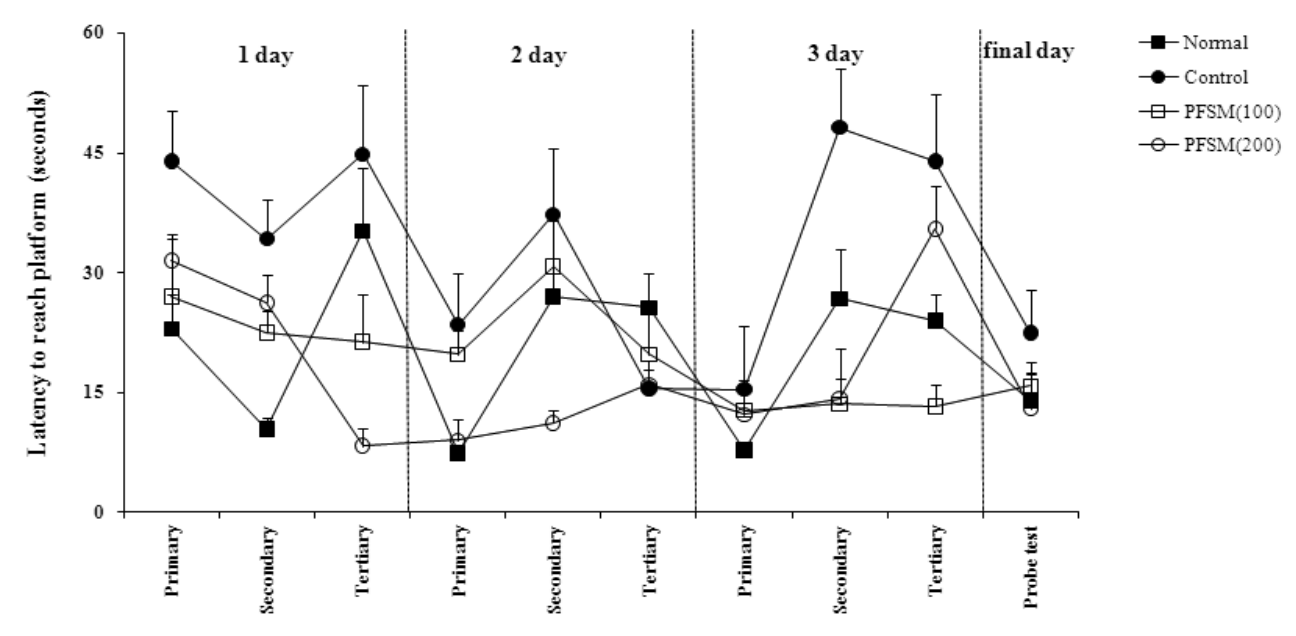

Figure 4. Effects of extract from purple sweet potato added to Bacillus subtilis-fermented soymilk on spatial learning and memory impairment in mice injected with $A \beta_{25-35}$ in Morris water maze tests. Values are mean \pm SD. Abbreviations were shown in Table 1

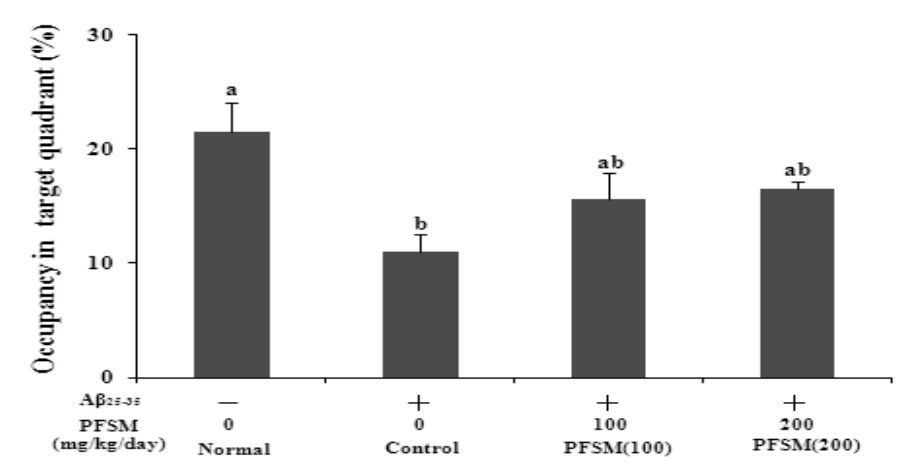

Figure 5. Effects of extract from purple sweet potato added to Bacillus subtilis-fermented soymilk on the performance of $A \beta_{25-35}$ treated mice at the former platform position in the transfer test of the Morris water maze test. Values are mean $\pm \mathrm{SD} .{ }^{\mathrm{a}-\mathrm{b}}$ Means with different letters are significantly different $(\mathrm{p}<0.05)$ by Duncan's multiple range test. Abbreviations are shown in Fig. 2.

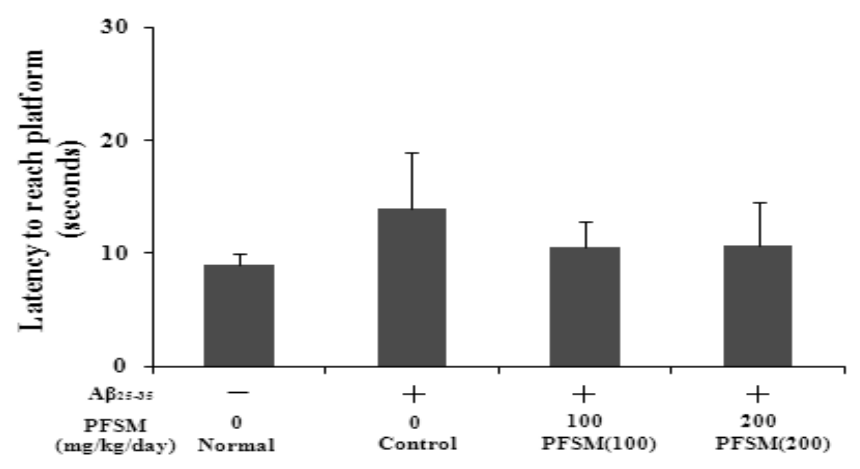

Figure 6. Effects of extract from purple sweet potato added to Bacillus subtilis-fermented soymilk on the performance of $A \beta_{25-35}$ treated mice in finding the exposed platform of the Morris water maze test. Values are means $\pm \mathrm{SD}$ 